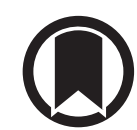

CrossMark

\title{
Xpert MTB/RIF as add-on test to microscopy in a low tuberculosis incidence setting
}

To the Editor:

Tuberculosis (TB) is a major public health concern worldwide. Early diagnosis, universal access to drug susceptibility testing and prompt initiation of treatment are key elements of the End TB strategy, and should therefore be implemented in all settings [1-5]. In order to reach TB elimination goals, the World Health Organization (WHO) currently recommends the use of a rapid molecular test, Xpert MTB/Rif (Xpert; Cepheid, Sunnyvale, CA, USA), as initial diagnostic tool when TB is suspected [6-8]. Although the excellent performance of this test in high TB burden areas is already supported by strong scientific evidence, few studies have been conducted so far to assess its impact on the diagnostic work-up of TB in low burden settings, sometimes with contrasting findings [7, 9, 10-12]. For example, according to SoHN et al. [10], Xpert testing might have limited impact in the ambulatory setting in Canada, owing to lower sensitivity and limited potential to expedite diagnosis beyond what is achieved with the existing, well-performing diagnostic algorithm.

We conducted a retrospective cohort study to assess the role of Xpert as add-on test to microscopy for TB diagnosis in a large metropolitan hospital of a medium-sized city of Northern Italy where the cumulative incidence of notified TB was 14.3 per 100000 in 2015 (i.e. approximately two-fold the national rate). The study was conducted in the units of infectious diseases, respiratory diseases and respiratory endoscopy. Our primary objective was to calculate the time to treatment initiation with and without Xpert testing, to assess the impact of this tool on clinical decision-making in a plausible context. We also aimed to determine the positive and negative predictive values (PPV and NPV) of Xpert compared to smear microscopy against the reference standard (culture), in patients with presumptive TB, i.e. those for whom clinicians requested laboratory tests to detect $M$. tuberculosis. All patients evaluated at study sites who had at least one biological sample investigated for TB between March 1, 2016 and June 30, 2017 were included. Sociodemographic, clinical and microbiological data were extracted from digital and paper clinical charts, and from laboratory archives. We defined as pulmonary TB cases all subjects with a positive culture for M. tuberculosis from a respiratory sample (i.e. sputum, bronchial aspirate, bronchoalveolar lavage). All anonymised data were retrospectively retrieved. Since our study had no impact on the patients' clinical management, ethical clearance was not required.

A total of 1201 cases were included in the analysis, that contributed with 1067 respiratory samples and 205 non-respiratory samples. Among 1067 patients with pulmonary disease, 79 (7.4\%) had microbiologically confirmed pulmonary TB. Only $26.3 \%(282 / 1067)$ of patients had at least one sample tested with Xpert: most of them were from infectious diseases unit (228/270, 84.4\%), compared to as few as $6.8 \%(54 / 797)$ of patients from either the respiratory diseases or respiratory endoscopy units $(\mathrm{p}<0.001)$.

Among 282 respiratory samples, Xpert was positive in 52/65 culture-positive cases and negative in 214/227 culture-negative cases. Sensitivity, specificity, PPV and NPV were 80\% (95\% CI 70.3-89.7\%), 98.6\% (95\% CI 96.1-100\%), 94.5\% (95\% CI 91.5-97.5\%), and 94.3\% (95\% CI 92.8-95.8\%), respectively.

Among 70 non-respiratory samples, Xpert was positive in 18/23 culture-positive cases and negative in 43/ 47 culture-negative cases. Sensitivity, specificity, PPV and NPV were 78.3\% (95\% CI 61.5-95.1\%), 91.5\% (95\% CI 83.5-99.5\%), 81.8\% (95\% CI 73.6-90\%), and 89.6\% (95\% CI 85.2-94\%) respectively.

@ERSpublications

Xpert MTB/Rif should be used as an alternative test for microscopy for TB diagnosis in low incidence settings http://ow.ly/JQCF30i8nO3

Cite this article as: Sulis G, Agliati A, Pinsi G, et al. Xpert MTB/RIF as add-on test to microscopy in a low tuberculosis incidence setting. Eur Respir J 2018; 51: 1702345 [https://doi.org/10.1183/ 13993003.02345-2017]. 
Among 65 culture-confirmed PTB cases with available microscopy and Xpert results, 43 (66.2\%) were smear-positive Xpert-positive, nine (13.8\%) smear-negative Xpert-positive, 13 (20\%) smear-negative Xpert-negative. Xpert increased the proportion of cases diagnosed with a rapid test by $21 \%(9 / 43)$. As expected, there were no smear-positive Xpert-negative tests among confirmed TB cases. The mean \pm SD time lag between sample collection and treatment initiation was 1.6 \pm 3.4 days for 47 smear-positive Xpert-positive, $1.8 \pm 2.1$ days for nine smear-negative Xpert-positive, and $8.7 \pm 8.8$ days for 13 smear-negative Xpert-negative patients $(\mathrm{p}<0.0001$ for the difference between the latter group and each of the two other groups) (table 1). The mean time to treatment initiation in microscopy-negative Xpert-negative cases remained shorter than the time needed for culture to become positive (mean \pm SD of $17 \pm 16$ days), showing that treatment was usually initiated empirically.

Among 205 patients with extrapulmonary disease, 38 (18.5\%) were culture-confirmed. Of the 23 individuals for whom both microscopy and Xpert results were available, eight (34.8\%) were smear-positive, while $10(43.5 \%)$ were smear-negative and Xpert-positive: Xpert led to a $125 \%$ gain in the proportion of extrapulmonary TB cases diagnosed with a rapid test. The mean \pm SD time lag between sample collection and treatment initiation was 3.0 \pm 5.4 days for eight smear-positive Xpert-positive, $1.5 \pm 2.0$ days for 10 smear-negative Xpert-positive, and 3.6 \pm 6.9 days for five smear-negative Xpert-negative patients (no statistically significant differences at an alpha level of 0.05 using a parametric test).

There were three cases of multidrug-resistant TB (2.9\%), all correctly identified by Xpert.

In low TB incidence, high resource countries the use of Xpert in clinical practice is not determined by the procurement capacity, but rather by the evidence of cost-benefit analyses. In agreement with previous studies [11], we found $14 \%$ additional rapid diagnoses of pulmonary $\mathrm{TB}$ with Xpert compared to microscopy, allowing treatment to commence about 7 days earlier. The gain was much larger for extrapulmonary TB cases (+125\%). In a German hospital, implementing Xpert as an add-on test in TB suspects resulted in cost savings [13]. Others had previously shown that Xpert could greatly reduce the frequency and impact of unnecessary empirical treatment and contact investigation [14]. Moreover, according to a study conducted in the USA, a single negative Xpert result is predictive of the absence of smear-positive/culture-positive tuberculosis with an NPV of $99.7 \%$, suggesting a role in removing patients from an airborne infection isolation room [15].

Xpert use was limited to about a quarter of presumptive TB cases, with pulmonary care units making very low use of the test. Even at the infectious diseases unit, Xpert was ordered in less than $50 \%$ of presumptive extrapulmonary $\mathrm{TB}$ cases, despite the proven benefits of this tool. Xpert scale-up will require intensive stewardship on TB diagnostic practices in all units where patients with presumptive TB are likely to be evaluated.

TABLE 1 Mean time to treatment initiation in patients included in the study (with and without culture-confirmed tuberculosis (TB)), classified by smear microscopy and Xpert MTB/RIF results

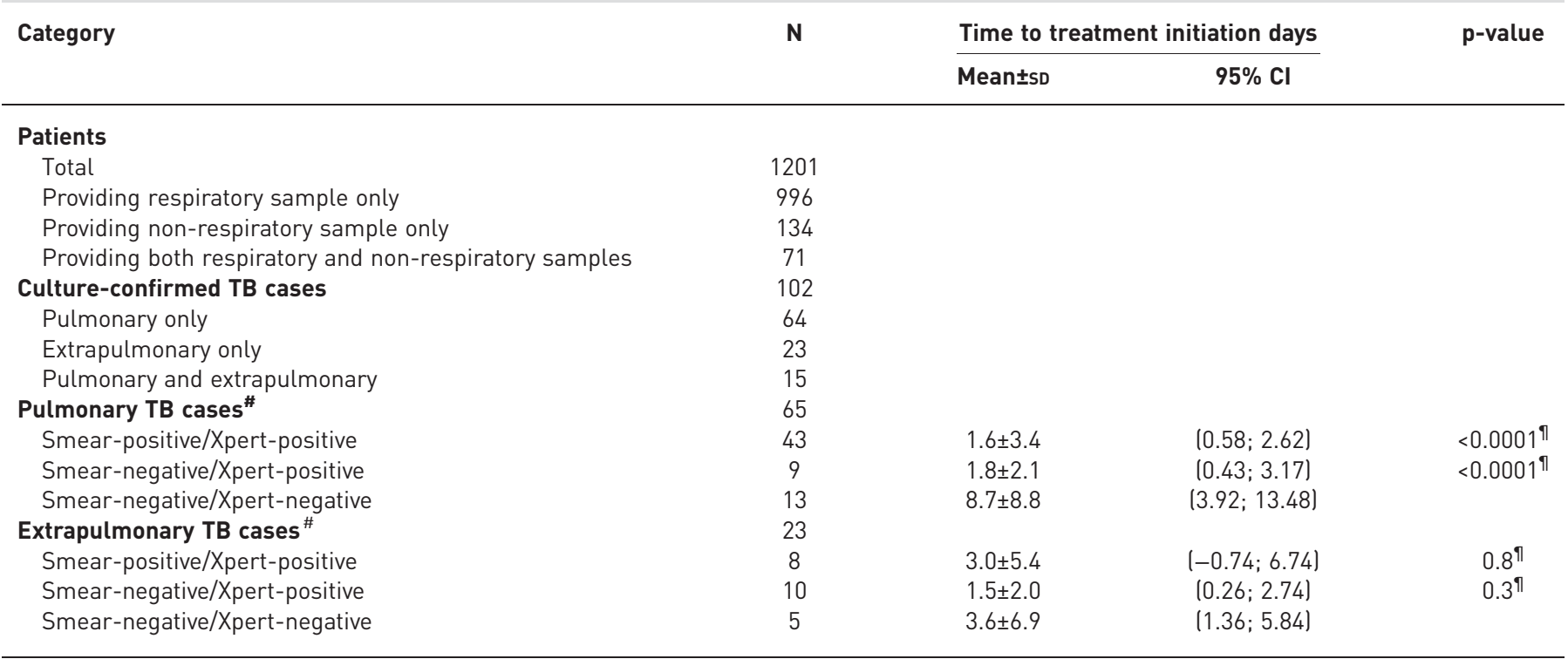

\#: with information on Xpert and time to treatment; ": two-tailed; compared with smear-negative/Xpert-negative. 
Our research has limitations: a low proportion of cases was investigated by Xpert, so we cannot exclude biases in the selection of samples that were submitted for testing. Moreover, we did not measure important outcomes, such as the effect of Xpert results on empirical TB treatment and on isolation practices, and we did not perform a cost-benefit analysis.

In conclusion, we provide an estimate of the benefits of Xpert compared to microscopy in the diagnosis of pulmonary and extrapulmonary TB in a low incidence setting. According to our study, Xpert plays an important role in guiding the clinicians' decision towards a prompt initiation of TB therapy even in a low burden setting, thus allowing for a greater number of potentially infectious patients to be rapidly put on treatment while waiting for culture results.

These data favour the use of Xpert as an alternative, rather than as add-on test to microscopy, in agreement with WHO recommendations [6].

Giorgia Sulis $\circledast^{1,2}$, Angelica Agliati ${ }^{1}$, Gabriele Pinsi ${ }^{3}$, Giordano Bozzola ${ }^{4}$, Pierfranco Foccoli ${ }^{5}$, Maurizio Gulletta ${ }^{6}$, Silvio Caligaris ${ }^{6}$, Lina R. Tomasoni ${ }^{6}$, Issa El-Hamad ${ }^{1}$ and Alberto Matteelli ${ }^{1}$

${ }^{1}$ Unit of Infectious Diseases, Dept of Clinical and Experimental Sciences, University of Brescia, Brescia, Italy. ${ }^{2}$ Dept of Epidemiology, Biostatistics and Occupational Health, McGill International TB Center, McGill University, Montreal, QC, Canada. ${ }^{3}$ Laboratory of Microbiology, Spedali Civili General Hospital, Brescia, Italy. ${ }^{4}$ Unit of Respiratory Diseases, Spedali Civili General Hospital, Brescia, Italy. ${ }^{5}$ Unit of Respiratory Endoscopy, Spedali Civili General Hospital, Brescia, Italy. ${ }^{6}$ Unit of Tropical Diseases, Spedali Civili General Hospital, Brescia, Italy.

Correspondence: Alberto Matteelli, Dept of Infectious and Tropical Diseases, University of Brescia and Spedali Civili General Hospital, P.le Spedali Civili, 1 25123, Brescia, Italy. E-mail: alberto.matteelli@unibs.it

Received: Nov 142017 | Accepted after revision: Jan 102018

Conflict of interest: None declared.

\section{References}

1 Voniatis C, Migliori GB, Voniatis M, et al. Tuberculosis elimination: dream or reality? The case of Cyprus. Eur Respir J 2014; 44: 543-546.

2 Lönnroth K, Migliori GB, Abubakar I, et al. Towards tuberculosis elimination: an action framework for low-incidence countries. Eur Respir J 2015; 45: 928-952.

3 Uplekar M, Weil D, Lonnroth K, et al. WHO's new end TB strategy. Lancet 2015; 385: 1799-1801.

4 Al Yaquobi F, Al-Abri S, Al Abri B, et al. TB elimination, dream or reality? The case of Oman. Eur Respir J 2018; 51: 1702027.

5 Blasi F, Matteelli A, Sotgiu G, et al. Moving towards tuberculosis elimination: a call for action from Italy and a possible model for other low tuberculosis incidence countries. Eur Respir J 2017; 49: 1702242.

6 World Health Organization (WHO). Automated Real-Time Nucleic Acid Amplification Technology for Rapid and Simultaneous Detection of Tuberculosis and Rifampicin Resistance: Xpert MTB/RIF Assay for the Diagnosis of Pulmonary and Extrapulmonary TB in Adults and Children: Policy Update. Geneva, World Health Organization (WHO), 2013.

7 Albert H, Nathavitharana RR, Isaacs C, et al. Development, roll-out and impact of Xpert MTB/RIF for tuberculosis: what lessons have we learnt and how can we do better? Eur Respir J. 2016; 48: 516-525.

8 World Health Organization (WHO). Compendium of WHO guidelines and associated standards: ensuring optimum delivery of the cascade of care for patients with tuberculosis. Geneva, World Health Organization (WHO), 2017.

9 Denkinger CM, Schumacher SG, Boehme CC, et al. Xpert MTB/RIF assay for the diagnosis of extrapulmonary tuberculosis: a systematic review and meta-analysis. Eur Respir J 2014; 44: 435-446.

10 Sohn H, Aero AD, Menzies D, et al. Xpert MTB/RIF testing in a low tuberculosis incidence, high-resource setting: limitations in accuracy and clinical impact. Clin Infect Dis 2014; 58: 970-976.

11 Lombardi G, Di Gregori V, Girometti N, et al. Diagnosis of smear-negative tuberculosis is greatly improved by Xpert MTB/RIF. PLoS One. 2017; 12: e0176186.

12 Rossato Silva D, Sotigu G, D'Ambrosio L, et al. Diagnostic performances of the Xpert MTB/RIF in Brazil. Respir Med 2018; 134: 12-15.

13 Diel R, Nienhaus A, Hillemann D, et al. Cost-benefit analysis of Xpert MTB/RIF for tuberculosis suspects in German hospitals. Eur Respir J 2016; 47: 575-587.

14 Davis JL, Kawamura LM, Chaisson LH, et al. Impact of GeneXpert MTB/RIF on patients and tuberculosis programs in a low-burden setting. A hypothetical trial. Am J Respir Crit Care Med 2014; 189: 1551-1559.

15 Luetkemeyer AF, Firnhaber C, Kendall MA, et al. Evaluation of Xpert MTB/RIF versus AFB smear and culture to identify pulmonary tuberculosis in patients with suspected tuberculosis from low and higher prevalence settings. Clin Infect Dis 2016; 62: 1081-1088. 